\title{
Doppler echocardiography evaluation of the CarboMedics valve in patients with small aortic anulus and valve prosthesis-body surface area mismatch
}

\begin{abstract}
A Doppler echocardiographic study was performed to evaluate the hemodynamic performances of small diameter CarboMedics aortic valves (CarboMedics, Inc., Austin, Tex.) in patients with a mismatch between the prosthetic valve and body surface area. Fourteen patients receiving either a $19 \mathrm{~mm}$ $(n=7)$ or a $21 \mathrm{~mm}$ valve $(n=7)$ prosthesis were studied. Only patients with a body surface area greater than $1.65 \mathrm{~m}^{2}$ were included in the study. Pulsed and continuous wave Doppler echocardiography was performed at rest and 2 minutes after treadmill exercise with the Bruce protocol. Peak and mean gradients across the valve prosthesis were estimated; effective orifice area, performance index, and discharge coefficient of the valve prosthesis were calculated. All patients achieved a significant increase in heart rate, systolic blood pressure, and cardiac output with exercise. Mean gradients \pm standard deviation of the mean at rest and 2 minutes after exercise were $20.1 \pm 7.1 \mathrm{~mm}$ $\mathrm{Hg}$ and $21.8 \pm 9 \mathrm{~mm} \mathrm{Hg}$ for the $19 \mathrm{~mm}$ prosthesis and $12.3 \pm 3.4 \mathrm{~mm} \mathrm{Hg}$ and $15.9 \pm 3.9 \mathrm{~mm} \mathrm{Hg}$ for the $21 \mathrm{~mm}$ prosthesis. The $19 \mathrm{~mm}$ valve prosthesis significantly increased the effective orifice area with exercise $(1.02 \pm 0.2$ versus $1.20 \pm 0.3 ; p<0.05)$, whereas it was almost unmodified for the 21 $\mathrm{mm}$ valve (1.38 \pm 0.2 versus $1.39 \pm 0.3 ; p=$ not significant). Therefore, despite a similar increase in cardiac output with exercise, only the $21 \mathrm{~mm}$ valve prosthesis showed a significant increase in peak $(25.4 \pm 5.2$ versus $34.9 \pm 8.1 \mathrm{~mm} \mathrm{Hg})$ and mean gradient $(p<0.01)$. We conclude that small diameter CarboMedics valves have satisfactory hemodynamic performances even after strenuous exercise in patients with large body surface areas. The hemodynamic performances of the $19 \mathrm{~mm}$ valve prosthesis seem to be optimized with exercise. (J ThORAC CardolovasC SuRG 1994;108:57-62)
\end{abstract}

Ruggero De Paulis, MD, Luigi Sommariva, MD, Fabrizio Russo, MD, Fabrizio Tomai, MD, Antonio Tondo, MD, Carlo Pagliaricci, MD, Carlo Bassano, MD, and Luigi Chiariello, MD, Rome, Italy

$\mathrm{T}_{\text {he }}$ he CarboMedics aortic valve prosthesis (CarboMedics, Inc., Austin, Tex.) is a bileaflet valve incorporating several design modifications of existing bileaflet valves that should result in improved performances. Among

From the Department of Cardiac Surgery, University of Rome, Tor Vergata, Rome, Italy.

Received for publication June 28, 1993.

Accepted for publication Oct. 17, 1993.

Address for reprints: Ruggero De Paulis, MD, Cattedra di Cardiochirurgia, Universita di Roma, Tor Vergata, European Hospital, via Portuense 694, 00149 Roma, Italy.

Copyright ${ }^{(\mathcal{1}} 1994$ by Mosby-Year Book, Inc.

$0022-5223 / 94 \$ 3.00+0 \quad \mathbf{1 2} / \mathbf{1} / \mathbf{5 2 3 1 2}$ these improved designs is the elimination of pivot guards, struts, and orifice projection, which is thought to reduce turbulence, and a reduced leaflet excursion that should ensure a more rapid and synchronous opening and closure. The plane of each leaflet forms angles of 25 and 78 degrees relative to the plane of the orifice ring in the closed and open position, respectively.

To evaluate the hemodynamic performances of small diameter CarboMedics prosthetic valves, we studied a selected patient population with a relatively great body surface area (BSA) and a small aortic anulus by means of Doppler echocardiography at rest and after isotonic exercise. 
Table I. Patient characteristics

\begin{tabular}{lccc}
\hline & \multicolumn{2}{c}{ Valve size } & \\
\cline { 2 - 3 } & $19 \mathrm{~mm}$ & $21 \mathrm{~mm}$ & p Value \\
\hline Age (yr) & $68.1 \pm 3.6$ & $66.2 \pm 9.1$ & $\mathrm{NS}$ \\
Follow-up time (mo) & $13 \pm 5.8$ & $10.2 \pm 5.6$ & $\mathrm{NS}$ \\
BSA (m $\left.{ }^{2}\right)$ & $1.73 \pm 0.06$ & $1.78 \pm 0.08$ & $\mathrm{NS}$ \\
$\mathrm{CO}(\mathrm{L} / \mathrm{min}) 2$ & $7.6 \pm 0.7$ & $7.6 \pm 2.2$ & $\mathrm{NS}$ \\
$\quad \begin{array}{l}\text { minutes after } \\
\quad \text { exercise }\end{array}$ & & & \\
HR 2 minutes after & $105 \pm 11.6$ & $106 \pm 12.6$ & $\mathrm{NS}$ \\
$\quad$ exercise & & & \\
$\begin{array}{l}\text { SPB (mm Hg) 2 } \\
\quad \text { minutes after }\end{array}$ & $136 \pm 14$ & $145 \pm 20$ & $\mathrm{NS}$ \\
$\quad$ exercise
\end{tabular}

All values are expressed as mean \pm standard deviation. $H R$, Heart rate; $S B P$, systolic blood pressure.

\section{Patients and methods}

Patient population. The study population comprised 14 consecutive patients (four men, ten women) ranging from 54 to 83 years of age (mean $67.2 \pm 6.7$ years) who underwent aortic valve replacement with a CarboMedics bileaflet valve prosthesis between January 1991 and January 1993. Only patients with a BSA greater than $1.65 \mathrm{~m}^{2}$ were included in the study. Seven of them received a $19 \mathrm{~mm}$ valve prosthesis, and seven had a 21 $\mathrm{mm}$ valve prosthesis. Five patients underwent valve replacement for severe aortic stenosis, two for valve regurgitation, and the remaining seven for mixed lesions. In two cases a mitral commissurotomy was associated, and in two cases a single coronary bypass was required. The interval between surgical intervention and follow-up Doppler evaluation ranged from 2 to 24 months (mean 11.6 \pm 5.6 ). All patients were free of symptoms at follow-up examination and were in New York Heart Association class I or II. Medical ethics committee approval and informed patient consent for participation to the study was obtained in all cases. Patient characteristics were similar in both valve size groups and are reported in Table I.

Study protocol. Echocardiography was performed with a Hewlett-Packard model 77025A echocardiograph with a 2.0 to 2.5 MHz transducer (Hewlett-Packard, Andover, Mass.) with facilities for pulsed wave Doppler and a second transducer for continuous wave Doppler echocardiography. Doppler studies were performed at rest and 2 minutes after treadmill exercise testing with the Bruce protocol. Flow velocity across the valve was measured by means of continuous wave Doppler echocardiography from three views-apical, right parasternal, and suprasternal-to record the highest transvalvular velocities. The ultrasonography window from which the highest velocities were obtained was selected and used for Doppler evaluation at rest and 2 minutes after exercise. The peak velocity was obtained, averaging from three to ten velocity envelopes for patients in sinus rhythm or in atrial fibrillation, respectively. The modified Bernoulli equation was used to calculate the instantaneous pressure gradients across the prosthesis. The velocity in the left ventricular outflow tract (LVOT) was considered in the gradient calculation by including it in the equation. The mean pressure gradient was calculated by averaging the gradient at 40 msec intervals throughout the velocity complexes. All patients then performed a symptom-limited, computer-assisted (CASE 12; Marquette, Milwaukee, Wis.) treadmill exercise test with the Bruce protocol. The exercise was prolonged until at least $85 \%$ of the maximal age-predicted heart rate was reached. Continuous wave Doppler echocardiography was performed 2 minutes after exercise with the same modality as at rest. Velocities across the prosthesis were recorded 2 minutes after the end of exercise because at this time the peak flow velocity reaches its highest level. ${ }^{1}$ Gardin and associates ${ }^{1}$ also showed that flow velocity integral, an estimate of relative stroke volume multiplied by the heart rate, is still $90 \%$ above control values during early recovery ( 2 minutes). Moreover, 2 minutes are also necessary to reobtain the best Doppler-beam flow alignment as stated by Kenny and associates ${ }^{2}$ who used a similar protocol. Arterial blood pressure (cuff sphygmomanometer) and heart rate were obtained during baseline, at peak, and after exercise.

\section{Doppler measurements and calculations}

Effective orifice area. The effective orifice area (EOA) of the valve was calculated with the continuity equation by the simplified peak velocity method as EOA $=\mathrm{CSA}\left(\mathrm{Pk} \mathrm{V}_{\mathrm{LVOT}} / \mathrm{Pk} \mathrm{V}_{\text {jet }}\right)$, where CSA is the cross-sectional area of the LVOT, and Pk $\mathrm{V}_{\mathrm{LVOT}}$ and $\mathrm{Pk} \mathrm{V}_{\text {jet }}$ are the maximum velocity in the LVOT and across the valve, respectively. This simplified method has shown an extremely good correlation with that of the original continuity equation. ${ }^{3}$ Furthermore, cross-sectional area of the LVOT was determined assuming that the sewing ring of the valve is the cross-sectional area of the LVOT. This eliminates the variability of the various measurements and should be the method of choice when the valve size is known. ${ }^{3}$

Effective area index. The effective area index (EAI) that is a measure of how well the effective orifice area of the valve matches the BSA was calculated as EAI = EOA/BSA. This index was used as a confirmation of a good patient selection, that is, the presence of a mismatch between valve size and body surface area. According to Dumesnil and associates ${ }^{4}$, it would appear that an indexed prosthetic valve area greater than 0.9 $\mathrm{cm}^{2} / \mathrm{m}^{2}$ would be a minimal requirement to minimize the postoperative gradient.

Performance index. The performance index is a measure of how effectively the external dimension of the valve is used in providing forward flow, normalized to the valve size, and it is defined as the effective orifice area divided by the sewing ring area. ${ }^{5}$ Most articles provide the performance index, making possible the comparison with other valve types.

Discharge coefficient. The discharge coefficient is calculated from the effective orifice area divided by the calculated orifice area and is a measure of how effectively a valve uses its flow area.

Cardiac output. The cardiac output (CO) was calculated using the formula $\mathrm{CO}=$ time velocity integral $\times$ effective orifice area $\times$ heart rate. ${ }^{6}$

All parameters were calculated independently by two expert echocardiographers. A third investigator reviewed the echocardiograms in blinded manner to the results if the first two investigators were not in agreement. The magnitude of the difference and its variability (two standard deviations) ${ }^{7}$ for maximum velocity values in patients with aortic valve prosthesis between interobserver and intraobserver agreement previously obtained in our laboratory were $0.028 \pm 0.34$ and $0.028 \pm 0.36 \mathrm{~m} / \mathrm{s}$, respectively.

Specifications of the CarboMedics valve. The CarboMedics valve is an all-pyrolytic carbon bileaflet valve. The valve size 
indicates the sewing ring diameter that is larger than the orifice diameter. The sewing ring area, the orifice diameter, the internal orifice area, and the calculated orifice area for the 19 and 21 mm valve prosthesis were obtained from the manufacturer and are reported in Table II. Because of the presence of the two metallic leaflets the calculated orifice area is smaller than that derived from the orifice diameter.

Statistical analysis. Two-factor repeated measures analysis of variance with repeated measures on one factor was used to compare Doppler echocardiographic parameters at rest and 2 minutes after exercise in the two groups. When significant differences were detected, pairwise comparisons were made by the Scheffé $F$ test. Comparisons of the remaining continuous variables between the two groups were performed by an unpaired Student's $t$ test. Data are expressed as mean \pm 1 standard deviation; $p$ values $<0.05$ were considered significant.

\section{Results}

During exercise, all patients reached at least $85 \%$ of the maximal age predicted heart rate, as stated in the study protocol. The mean exercise duration was $5.9 \pm 1.6$ minutes ranging from 3.3 to 9.1 minutes. Heart rate and systolic blood pressure significantly increased in all patients. A significant increase in $\mathrm{CO}$ with exercise was observed in all cases, and no significant differences in exercise parameters were found between patients receiving different valve sizes (Table I).

Doppler echocardiography findings at rest and after exercise are shown in Table III. Only trivial valve regurgitation, compatible with that reported by the manufacturer, and without clinical significance, was recorded in all patients. As expected, patients with a $19 \mathrm{~mm}$ prosthesis had higher mean and instantaneous peak gradient than did those with a $21 \mathrm{~mm}$ prosthesis. However, despite a similar increase in $\mathrm{CO}$ with exercise, the proportional increase in flow velocity across the valve and therefore the gradient was significantly inferior for the $19 \mathrm{~mm}$ valve (Table III). The effective area index of both valves confirmed the inadequacy of effective orifice area for each BSA (Table III). The effective orifice areas in the resting condition were slightly inferior to the theoretical values reported from the manufacturer $\left(1.02\right.$ versus $1.06 \mathrm{~cm}^{2}$ for the $19 \mathrm{~mm}$ and 1.38 versus $1.41 \mathrm{~cm}^{2}$ for the $21 \mathrm{~mm}$ prosthesis, respectively) (Tables II and III); with exercise, however, a significant increase in effective orifice area was evident for the $19 \mathrm{~mm}$, whereas it was almost unmodified for the $21 \mathrm{~mm}$ prosthesis. This finding was also reflected by the performance index which was similar after exercise for the two valve sizes.

\section{Discussion}

Doppler ultrasonography has shown to be a dependable method to measure flow velocity and to estimate pressure
Table II. Specifications of the CarboMedics valve as obtained from the manufacturer

\begin{tabular}{lcc}
\hline & \multicolumn{2}{c}{ Valve size } \\
\cline { 2 - 3 } & $19 \mathrm{~mm}$ & $21 \mathrm{~mm}$ \\
\hline Anulus diameter $(\mathrm{mm})$ & 19.8 & 21.8 \\
Orifice diameter $(\mathrm{mm})$ & 14.7 & 16.7 \\
Anulus area $\left(\mathrm{cm}^{2}\right)$ & 3.07 & 3.73 \\
Internal orifice area $\left(\mathrm{cm}^{2}\right)$ & 1.59 & 2.07 \\
Calculated orifice area $\left(\mathrm{cm}^{2}\right)$ & 1.06 & 1.41 \\
\hline
\end{tabular}

gradients across valve prostheses. Sager and associates ${ }^{8}$ have found a significant correlation between mean Doppler and catheterization gradients in patients with Hancock (Johnson \& Johnson Cardiovascular, King of Prussia, Pa.) or Björk-Shiley (Shiley, Inc., Irvine, Calif.) aortic prostheses. More recently, in a study conducted in patients with different biologic and mechanical valves, Burstow and associates ${ }^{9}$ confirmed the correlation $(r=0.94)$ between Doppler measurements and those obtained with simultaneous catheterization of the left ventricular and aortic chambers, thus concluding that Doppler echocardiography can be reliably used for the measurement of prosthetic gradients. Central-flow aortic prosthesis, like the St. Jude Medical valve (St. Jude Medical, Inc., St. Paul, Minn.) and all kinds of biologic valves, should theoretically allow accurate Doppler evaluation as do native aortic valves. However, although this has been demonstrated for normal and stenotic biologic valves, ${ }^{10,11}$ it may not be true for bileaflet mechanical valves. Baumgartner and associates ${ }^{12}$ demonstrated discrepancies between Doppler and catheter gradients in the St. Jude Medical prosthetic valves; Doppler gradients were always higher because of the presence of high localized pressure gradients within the valve and a significant pressure recovery after blood exits the valve. Because the CarboMedics valve is a bileaflet mechanical valve with characteristics similar to those of the St. Jude Medical valve, the same conclusion should apply. In fact, a recent clinical study comparing invasive and noninvasive techniques for the CarboMedics valve in aortic position confirmed that mean Doppler gradients exceeded mean catheter gradients by $4.3 \pm 4.8 \mathrm{~mm} \mathrm{Hg}{ }^{13}$ We should therefore consider that the Doppler-defined gradients of our study could be a slight overestimation of catheter-derived gradients.

Another important point that needs to be taken into account is the problem of valve-patient mismatch. Jones and associates ${ }^{14}$ evaluated the hemodynamic and clinical performances of standard Hancock bioprosthesis and recommended never to use the $19 \mathrm{~mm}$ prosthesis and to 
Table III. Hemodynamic and echocardiographic findings before and 2 minutes after exercise in patients with 19 or $21 \mathrm{~mm}$ CarboMedics aortic valve

\begin{tabular}{|c|c|c|c|c|}
\hline & \multicolumn{2}{|c|}{$19 \mathrm{~mm}$ valve } & \multicolumn{2}{|c|}{$21 \mathrm{~mm}$ valve } \\
\hline & Rest & Exercise & Rest & Exercise \\
\hline $\mathrm{CO}(\mathrm{L} / \mathrm{min})$ & $4.8 \pm 0.5$ & $7.6 \pm 0.7^{*}$ & $5.6 \pm 1.2$ & $7.6 \pm 2.2^{*}$ \\
\hline Maximum gradient $(\mathrm{mm} \mathrm{Hg})$ & $33.4 \pm 13.2$ & $34.3 \pm 14.5$ & $25.4 \pm 5.2$ & $34.9 \pm 8.1^{*}$ \\
\hline Mean gradient $(\mathrm{mm} \mathrm{Hg})$ & $20.1 \pm 7.1$ & $21.8 \pm 9$ & $12.3 \pm 3.4$ & $15.9 \pm 3.9^{*}$ \\
\hline $\operatorname{EOA}\left(\mathrm{cm}^{2}\right)$ & $1.02 \pm 0.2$ & $1.20 \pm 0.3^{* *}$ & $1.38 \pm 0.2$ & $1.39 \pm 0.3$ \\
\hline $\operatorname{EAI}\left(\mathrm{cm}^{2}\right)$ & $0.59 \pm 0.1$ & $0.70 \pm 0.1^{* *}$ & $0.77 \pm 0.1$ & $0.78 \pm 0.2$ \\
\hline PI $\left(\mathrm{cm}^{2}\right)$ & $0.33 \pm 0.08$ & $0.40 \pm 0.1^{* *}$ & $0.37 \pm 0.1$ & $0.37 \pm 0.1$ \\
\hline $\mathrm{Cd}\left(\mathrm{cm}^{2}\right)$ & $0.96 \pm 0.2$ & $1.1 \pm 0.3^{* *}$ & $0.98 \pm 0.2$ & $0.99 \pm 0.2$ \\
\hline
\end{tabular}

All values are expressed as mean \pm standard deviation. $E O A$, Effective orifice area; $E A I$, effective area index; $P I$, performance index; $C d$, discharge coefficient. ${ }^{*} p<0.01$ versus resting values.

$*_{p} p<0.05$ versus resting values.

use the $21 \mathrm{~mm}$ only in very small patients. Schaff and associates ${ }^{15}$ invasively evaluated the $19 \mathrm{~mm}$ Björk-Shiley valve concluding that it gives satisfactory hemodynamic performances provided that BSA is less than $1.7 \mathrm{~m}^{2}$. Finally, Wortham and associates ${ }^{16}$ evaluated the 19 and $21 \mathrm{~mm}$ St. Jude Medical valve prostheses, suggesting that the use of the $19 \mathrm{~mm}$ size should be restricted to patients with a BSA less than $1.8 \mathrm{~m}^{2}$; however, the average BSA of their patients was only $1.49 \mathrm{~m}^{2}$. In our selected series only two patients had a BSA less than $1.70 \mathrm{~m}^{2}$, and the effective area indexes were extremely low ( 0.59 and 0.77 $\mathrm{cm}^{2}$ for 19 and $21 \mathrm{~mm}$ prosthesis, respectively). These findings testify to the important mismatch between flow area and BSA in our patients. By comparison in a study by Ihlen and associates, ${ }^{13}$ who used the same index, the effective area index for CarboMedics valve in aortic position was 0.72 and $0.89 \mathrm{~cm}^{2}$ for the valve sizes 19 and 21 $\mathrm{mm}$, respectively.

Therefore, considering the possibility of a gradient overestimation by the Doppler technique in this type of valve and the presence of valve prosthesis-BSA mismatch, our results seem to indicate that the CarboMedics valve is an acceptable device with favorable hemodynamic performances even after strenuous exercise. The effective flow areas at rest were similar to the in vitro theoretic values, thus showing that the hemodynamic potential is fully realized in vivo for both valve sizes. It is noteworthy that the discharge coefficient in the $19 \mathrm{~mm}$ valve prosthesis has the tendency to be greater with exercise, implying a more efficient use of the orifice area. This tendency is in contrast with the study of Gray and associates, ${ }^{17}$ which included a reduction of the discharge coefficient in St. Jude Medical valves after infusion of isoproterenol; this effect was ascribed to the turbulence occurring under conditions of higher flow. Because the discharge coefficient is an index of how effectively a valve uses its flow area, it is also an indirect measure of the effi- ciency of the moving parts of the valve as well as the optimization of the flow path across the prosthesis. Therefore, although the $19 \mathrm{~mm}$ valve has a low performance index as demonstrated by others ${ }^{13}$ and confirmed by us, the design of the internal area and the leaflets is optimized. We can therefore speculate, according to our results, that the hemodynamic performances of the smallest CarboMedics valve is optimized after exercise probably through a more rapid and synchronous opening of the two leaflets, thus limiting turbulence. Furthermore, the design of leaflets and the pivot and hinge mechanism seem to minimize the areas of flow separation and turbulent shear stress that would cause a decrease in discharge coefficient. However, further investigations are necessary to define the reason for the apparent superior performance during exercise of the $19 \mathrm{~mm}$ CarboMedics valve when compared with the $21 \mathrm{~mm}$ valve.

Our data are consistent with the few studies available in the literature reporting a Doppler echocardiographic evaluation of patients with the CarboMedics valve. Ihlen and associates ${ }^{13}$ reported maximal resting gradients of 30 and $21 \mathrm{~mm} \mathrm{Hg}$ for patients with 19 and $21 \mathrm{~mm}$ valve prosthesis respectively, whereas Globitz and associates ${ }^{18}$ found instantaneous peak gradients of 43 and $31 \mathrm{~mm} \mathrm{Hg}$, respectively. Our study also demonstrates that maximum and mean gradients increase only slightly with exercise, which shows the optimization of flow dymamics through the valve. Most studies evaluating the St. Jude Medical valve reported catheterization mean gradients varying from 7 to $14 \mathrm{~mm} \mathrm{Hg}$ for the $19 \mathrm{~mm}$ valve and an average of $5 \mathrm{~mm} \mathrm{Hg}$ for the $21 \mathrm{~mm}$ valve. ${ }^{17,}{ }^{19}$ Similar values were reported on the catheterization-derived gradients for the CarboMedics valve. ${ }^{13}$ However, although the hemodynamic performances of the two valves are comparable, the performance indexes found in our study, as well as those reported by Ihlen and associates, ${ }^{13}$ are not higher than $0.40 \mathrm{~cm}^{2}$; the St. Jude Medical valve, on the other hand, 
always shows a performance index varying from 0.56 to $0.71 \mathrm{~cm}^{2}$ in different reports. ${ }^{17,19}$ Furthermore, a performance index between 0.37 and $0.44 \mathrm{~cm}^{2}$ has been reported for small Björk-Shiley valves, ${ }^{10,20}$ and a performance index of $0.53 \mathrm{~cm}^{2}$ has been reported for the $21 \mathrm{~mm}$ Medtronic Hall mechanical valve (Medtronic, Inc., Minneapolis, Minn.). ${ }^{21}$ This finding led to the conclusion that, although the CarboMedics valve can be defined as a successful construction demonstrated by an in vivo discharge index equal to 1 , when compared with most mechanical valves, it shows ineffective use of the total area of the prosthesis.

Conclusions and limitations of the study. Several investigators have shown the value of Doppler echocardiography in the hemodynamic assessment of prosthetic biologic or mechanical valves. Although still controversial, a good correlation with catheterization study has been demonstrated in several studies. ${ }^{8-13}$ The use of Doppler echocardiography has allowed us to obtain hemodynamic information after exercise more strenuous than would have been possible during catheterization.

A possible limitation in our measurements is that a reactive hypertrophy in the LVOT might develop in a certain number of elderly patients with aortic stenosis. Therefore, the gradient across the LVOT can be created not only by the prosthesis but also by the outflow tract hypertrophy. Although only five patients (three with the $19 \mathrm{~mm}$ and two with the $21 \mathrm{~mm}$ valve) had pure aortic valve stenosis, we can not rule out the possibility of a less accurate echocardiographic assessment in these patients.

Our study involved only 14 patients, seven for each group, and this could account for less accurate statistical conclusions. However, the majority of reports are related to a small group of patients especially because they need to be categorized according to valve type and size. In our study, a further limitation was that only patients with a small aortic anulus and large BSA were selected, thus greatly narrowing the number of suitable patients. However, the evaluation of this small but particular group of patients allowed us to conclude that this type of prosthesis can be safely used in most clinical cases. A reduction in the ratio between sewing ring valve area and effective orifice area seems desirable.

\section{REFERENCES}

1. Gardin JM, Kozlowski J, Dabestani A, et al. Studies of Doppler aortic flow velocity during supine bicycle exercise. Am J Cardiol 1986;57:327-32.
2. Kenny A, Woods J, Fuller CA, et al. Hemodynamic evalevaluuation of the Monostrut and spherical disc BjörkShiley aortic valve prosthesis with Doppler echocardiography. J THORAC CARdiovasc SuRg 1992;104:1025-8.

3. Chafizadeh ER, Zoghbi WA. Doppler echocardiographic assessment of the St. Jude Medical prosthetic valve in the aortic position using the continuity equation. Circulation 1991;83:213-23.

4. Dumesnil JG, Honos GN, Lemieux M, Beauchemin J. Validation and application of indexed aortic prosthetic valve areas calculated by Doppler echocardiography. J Am Coll Cardiol 1990;16:637-43.

5. Gabbay S, Mc Queen DM, Yellin EL, Frater RWM. In vitro hydrodynamic comparison of mitral valve prostheses. Circulation 1979;60(Suppl):I62-70.

6. Huntsman LL, Stewart DK, Barnes SR, Franklin SB, Colocousis JS, Hessel EA. Noninvasive Doppler determination of cardiac output in man: clinical validation. Circulation 1983;67:593-602.

7. Bland JM, Altman DG. Statistical methods for assessing agreement between two methods of clinical measurements. Lancet 1986;1:307-10.

8. Sager KB, Wann LS, Paulsen WHJ, Romhilt DW. Doppler echocardiographic evaluation of Hancock and Björk-Shiley prosthetic valves. J Am Coll Cardiol 1986;7: 681-7.

9. Burstow DJ, Nishimura RA, Bailey KR, et al. Continuous wave Doppler echocardiographic measurements of prosthetic valve gradients: a simultaneous Doppler-catheter study. Circulation 1989;80:504-14.

10. Reisner SA, Meltzer RS. Normal values of prosthetic valve Doppler echocardiographic parameters: a review. J Am Soc Echo 1988;1:201-10.

11. Rothbart RM, Castriz JL, Harding LV, Russo CD, Teague $\mathrm{SM}$. Determination of aortic valve area by two-dimensional and Doppler echocardiography in patients with normal and stenotic bioprosthetic valves. J Am Coll Cardiol 1990; 15:817-24.

12. Baumgartner $\mathrm{H}$, Khan $\mathrm{S}$, DeRobertis $\mathrm{M}$, Czer L, Maurer $\mathrm{G}$. Effect of prosthetic aortic valve design on the Dopplercatheter gradient correlation: an in vitro study of normal St. Jude, Medtronic-Hall, Starr-Edwards and Hancock valves. J Am Coll Cardiol 1992;19:324-32.

13. Ihlen $H$, Molstad P, Simonsen S, et al. Hemodynamic evaluation of the CarboMedics prosthetic heart valve in the aortic position: comparison of noninvasive and invasive techniques. Am Heart J 1992;123:151-9.

14. Jones EL, Craver JM, Morris DC, et al. Hemodynamic and clinical evaluation of the Hancock xenograft bioprosthesis for aortic valve replacement (with emphasis on the management of the small aortic root). J THORAC CARDIOVASC SURG 1978;75:300-8.

15. Schaff HV, Borkon AM, Hughes C, et al. Clinical and hemodynamic evaluation of the $19 \mathrm{~mm}$ Björk-Shiley aortic valve prosthesis. Ann Thorac Surg 1981;32:50-7.

16. Wortham DC, Tri TB, Bowen TE. Hemodynamic evalua- 
tion of the St. Jude Medical valve prosthesis in the small aortic anulus. J ThoraC CARdiovaSC SuRg 1981;81:61520.

17. Gray RJ, Chaux A, Matloff JM, et al, Bileaflet, tilting disc, and porcine aortic valve substitutes: in vivo hydrodynamics characteristics. J Am Coll Cardiol 1984;3:321-7.

18. Globitz S, Rodler S, Mayr H, et al. Doppler sonographic evaluation of the Carbomedics bileaflet valve prosthesis: one-year experience. J Cardiac Surg 1992;7:9-16.

19. Chaux A, Gray RJ, Matloff JM, Feldman H, Sustaita H. An appreciation of the new St. Jude valvular prosthesis. J Thorac Cardiovasc SuRg 1981;81:202-11.
20. Aberg B, Holmgren A. Hemodynamic evaluation of the Convexo-concave Björk-Shiley prosthesis in patients with narrow aortic annulus. Scand J Thorac Cardiovasc Surg 1981;15:111-6.

21. Nitter-Hauge S, Enge I, Sembe BKH, Hall KV. Primary clinical experience with the Hall-Kaster valve in the aortic position: results at three months including hemodynamic studies. Circualtion 1979;60(Suppl): I55-62. 